\title{
Lifestyle Ubiquitous Gaming: Computer Games Making Daily Lives Fun
}

\author{
Eiji Tokunaga, Masaaki Ayabe, Hiroaki Kimura, and Tatsuo Nakajima \\ Department of Information and Computer Science, Waseda University \\ 3-4-1 Okubo Shinjuku Tokyo 169-8555, Japan \\ Tel\&Fax:+81-3-5286-3185 \\ \{eitoku, ayabe, hiraoki, tatsuo\}@dcl.info.waseda.ac.jp
}

\begin{abstract}
We propose a novel computer gaming style in which users can enjoy games through their daily lives without paying too much attention and time for the games, we call it lifestyle ubiquitous gaming. Lifestyle ubiquitous games track human daily activities implicitly and incorporate the tracked activities in their game logics. Then they represent decorated and virtualized those activities on ambient displays. We believe that this gaming style enables not only making their boring and messy daily tasks fun but improving their lazy lifestyle and customs. In this paper, we describe our lifestyle ubiquitous gaming concept and framework, and then we show our two case studies implementing distinguishing scenarios.
\end{abstract}

\section{Introduction}

Recent computer game technologies enable us to play games anytime, anywhere and with anyone. The latest portable/non-portable game consoles have significant computing resources and network capabilities, and a lot of games are provided on the cellphone market. On the other hand, the purpose of playing computer games is becoming diverse and expanding. The positive effects of games are being explored in both of academic and industrial research. This is not only for entertainment but also for communication, education, health, business and even military.

In this way it is broadening the consumer base, however, there are still many ordinary people who cannot play computer games even they love playing games. Because current computer games have three significant negative factors to be played by non-gamers: 1) Most of current games require a lot of time to enjoy so that busy people cannot enjoy enough. In particular, massively multiplayer online games require a large amount of time to sufficiently enjoy in their virtual world. 2) Resent games are not only just too hard to enjoy, but also that requires unignorable physical work and psychological barrier to start playing them. We have to turn on the TV and game console, then switch the TV input to which the console is connected to play ordinary console games. Online games might require additional work. Even for mobile games, players must carry slightly heavy mobile game consoles and find appropriate places to play just games. 3) Playing computer games is still considered as socially disgraceful by certain people, even 
by players themselves. Some of Japanese female gamers like to play games using cell phones because other people cannot recognize they are playing computer games. But, it is still slightly strange that a person is giving a steady look into the display of her cell phone, and intensely pushing some buttons.

Therefore, we propose a novel computer gaming style in which gaming features are integrated into our daily lives seamlessly by using sensing technologies. The games extract real-time context and history of everyday activities such as cooking and cleaning. Then, the extracted daily activities are decorated with gaming features and represented in ambient style. Players do no have to be very conscious of how they are and other people are fairly ignorant of what they are doing there. They do not have to take time for games into account, just do daily activities augmented with fun games. They can enjoy and enhance their daily lives by gaming effects with very little efforts. In other words, it makes possible to learn by games for not science or physics but our daily lives. We describe one possible future scenario as follows.

SensPet: Eiji loves to play computer games very much but he is too busy to play recent Hollywood movie-like large-scale games. Nowadays, he is a huge fun of SensPet that is an online virtual fuss-free pet game. SensPet provides a virtual pet that is a virtual counter part of Eiji's daily life. Surrounding sensors in his home or mobile phone is sensing his daily activities, and then extracted contexts automatically influence the growth of his pet. If he cleans his room often, his pet house is always clean and his pet grows up elegant. If he buys fancy clothes, his pet is dressed up and grows up smart. Unlike other virtual pet games that require too much investment to breed, SensPet does not require much time and high skills to enjoy but exciting. If he is smart with daily activities, his pet grows up smart. He is strongly motivated to do the best for his daily life. The vender of SensPet will provide the next version of SensPet that is "SensCity" for intellectual people...

We call this kind of gaming style lifestyle ubiquitous gaimng. We believe that it enables not only making their boring and messy daily tasks fun but improving their lazy lifestyle and customs. Exploring this novel gaming style leads to establish new outpost for gaming industries. The rest of this paper is structured as follows. Section 2 describes the conceptual framework of lifestyle ubiquitous gaming. We built two lifestyle ubiquitous games on the framework as case studies. Section 3 describes the design and implementation of the case studies. Section 4 discusses some issues and expected exit. Section 5 shows related work and Section 6 concludes this paper with future work.

\section{Lifestyle Ubiquitous Gaming Framework}

Fig. 11 shows the overview of lifestyle ubiquitous gaming conceptual framework. This framework consists of four stages: lifestyle tracking, sensor analysis, game logic, lifestyle presentation enclosing human daily activities. These stages transit as a cycle as shown in the figure. We describe about the characteristic of each stage and human daily activities to be used in lifestyle ubiquitous gaming as follows. 


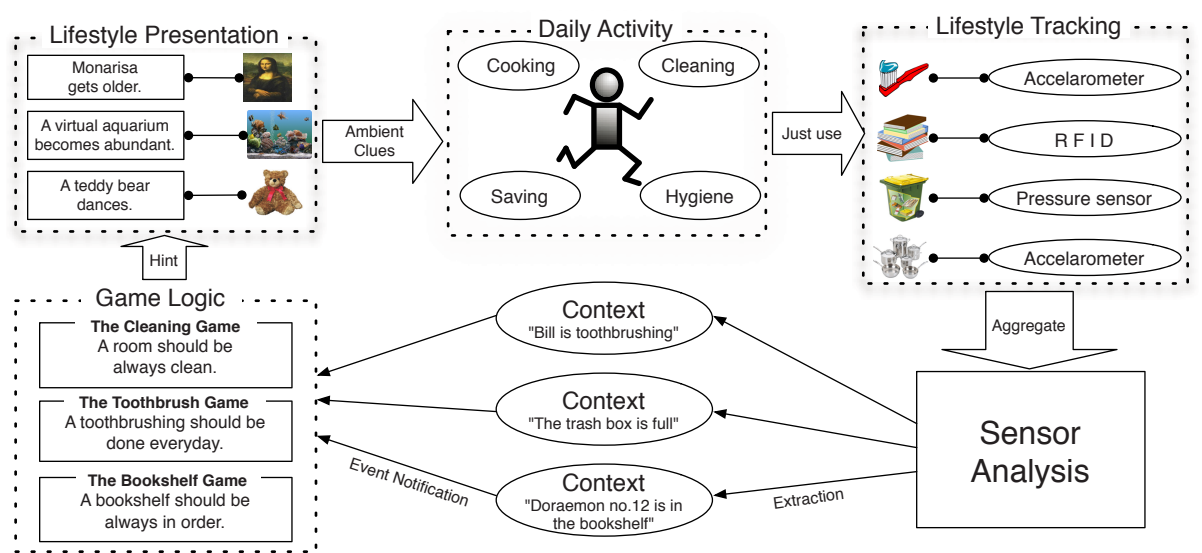

Fig. 1. Lifestyle ubiquitous gaming conceptual framework overview

Lifestyle tracking: In lifestyle ubiquitous gaming, players basically just use daily objects such as a toothbrush, a bookshelf and a kitchen knife in their regular daily lives. Their daily activities using the daily objects are tracked implicitly by sensors embedded in the daily objects [7]. The tracked data is passed to the next stages that use them as input events on the gaming world. In this way, players do not have to very conscious of playing games, and it is very easy to play lifestyle ubiquitous games for even non-gamers such as elderly people.

Sensor analysis: Each tracked data extracted from embedded sensors is often too fine-grained to use in game logics. This stage analyzes the tracked data into contexts that are highly abstracted activity information such as "Alice is brushing her teeth" and "The Art of Computer Programming is in the bookshelf".

Game logic: In this stage, goals/idempotents of lifestyle ubiquitous gaming such as making cooking fun and motivating daily cleaning are incorporated with analyzed contexts in game logics. For example, a logic motivating daily cleaning one's own room stores cleaning contexts of player's rooms daily and defines the states of their rooms such as "Alice's room is clean", "Bob's room is dirty" or "Carol's room is not so clean even she often cleans her room". It decides what kind of gaming representation would be shown for making their each cleaning activity fun and motivating them. Then it generates hints for achieving the goal/idempotent.

Lifestyle presentation: Unlike in the case of ordinary computer games, too blinging and bright representation would be annoying and disturbing in lifestyle ubiquitous gaming style. In this stage, the hints generated in the game logic stage are represented through daily representation objects such as a fine art, an aquarium and a teddy bear. These objects provide ambient clues to achieve goals of the games in restrained ways that do not require too much time and attention to 
understand. Those goals also mean, in other words, improving players' lives and making their custom better.

\subsection{Human Daily Activities}

Currently, we have selected four human daily activities to be augmented with games: cleaning, hygiene, saving, cooking. We considered possibilities for improvement about the activity and technological implementability to track them.

Cleaning activities: Although cleaning our living environments is necessary to live healthfully, it is boring task for many people. If we can augment the cleaning task with gaming, people who are lazy for cleaning might be decrease. Even for the people who are not lazy, the game would make a part of their daily lives amusing. We can know how clean a room is using dust sensors and movements of cleaners and sweepers.

Hygiene activities: Hygiene activities like tooth brushing and hand washing are obviously quite important for healthy daily lives. But, children and even adults often lazy for doing such hygiene activities. We would like to encourage the continuation of them by presenting the use of hygiene things such as toothbrushes and hand soaps in a virtual gaming world. We can know by whom and when the toothbrushes are used by attaching accelerometers to them [6]. We assume that users will not choose the wrong toothbrush.

Cooking activities Cooking and eating well-balanced meals is one of the most significant ingredients to improve our quality of life. However, managing the balance is not so easy for ordinary people, and its continuation is even more difficult. If we can build a game in which cooking well-balanced meals is connected to positive feedbacks, it might increase mothers motivation to cook well and encourage children to eat wel-balanced meals.

Resource saving activities Saving resources like electricity and water leads to save our money and help natural resources on the earth. But the respective resource saving activities in our daily lives, such as turning off rights and appliances regularly is often very hard to be aware as actually be connected to save resources. If a game can show how the saving activity effects to the overall goal and the total amount of saved resources in a fun way, they might continue to save resources without patience.

\section{Case Studies}

We built prototype games based on the described conceptual framework to confirm feasibility and effectivity of our lifestyle ubiquitous gaming concept. Currently, we have built two games one is for a kind of hygiene activities: toothbrushing, the another one is for a kind of cleaning activities: arranging a bookshelf. 


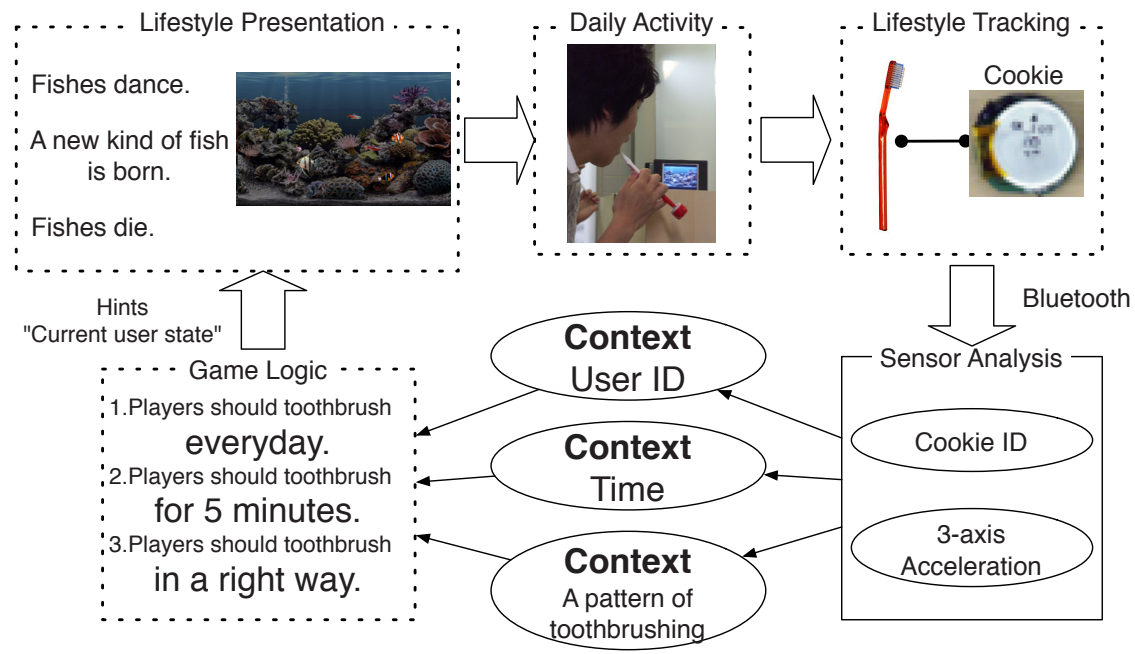

Fig. 2. Toothbrushing connected to breeding tropical fish

\subsection{A Virtual Aquarium Improving Toothbrushing Activity}

The daily activity in Fig. 2 shows a player toothbrushing, which is a kind of hygiene activities, in front of a virtual aquarium displayed on a micro pc. This game is based on three rules that are players should tooth brush everyday, players should toothbrush for 5 minutes and players should toothbrush in a right way. It tracks toothbrushing activity and provides immediate feedback for encouraging toothbrushing in sufficient time, and gradual feedback for encouraging continuance of everyday toothbrushing in a right way.

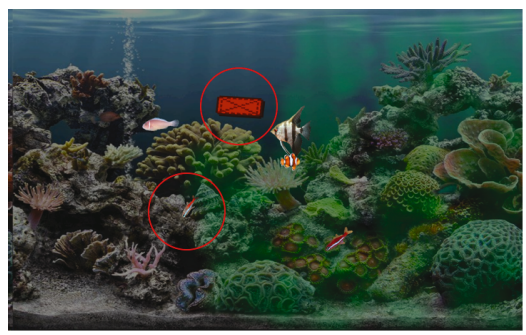

Fig. 3. Dancing fish and wiping rag while player's toothbrushing

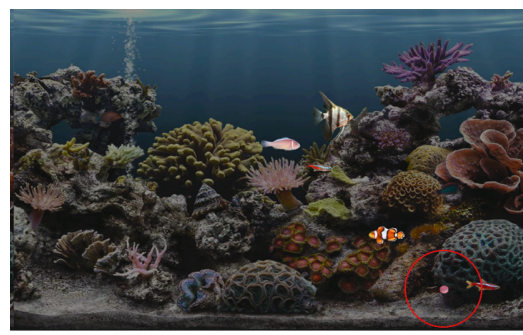

Fig. 4. A spawned egg after player's wellregulated toothbrushing

Immediate feedback: When a player starts toothbrushing, fish in the aquarium dance happily and a rag starts wiping a moss-covered window (Fig. 3i). Then, while s/he continues to brush in sufficient time, their dance changes into more 


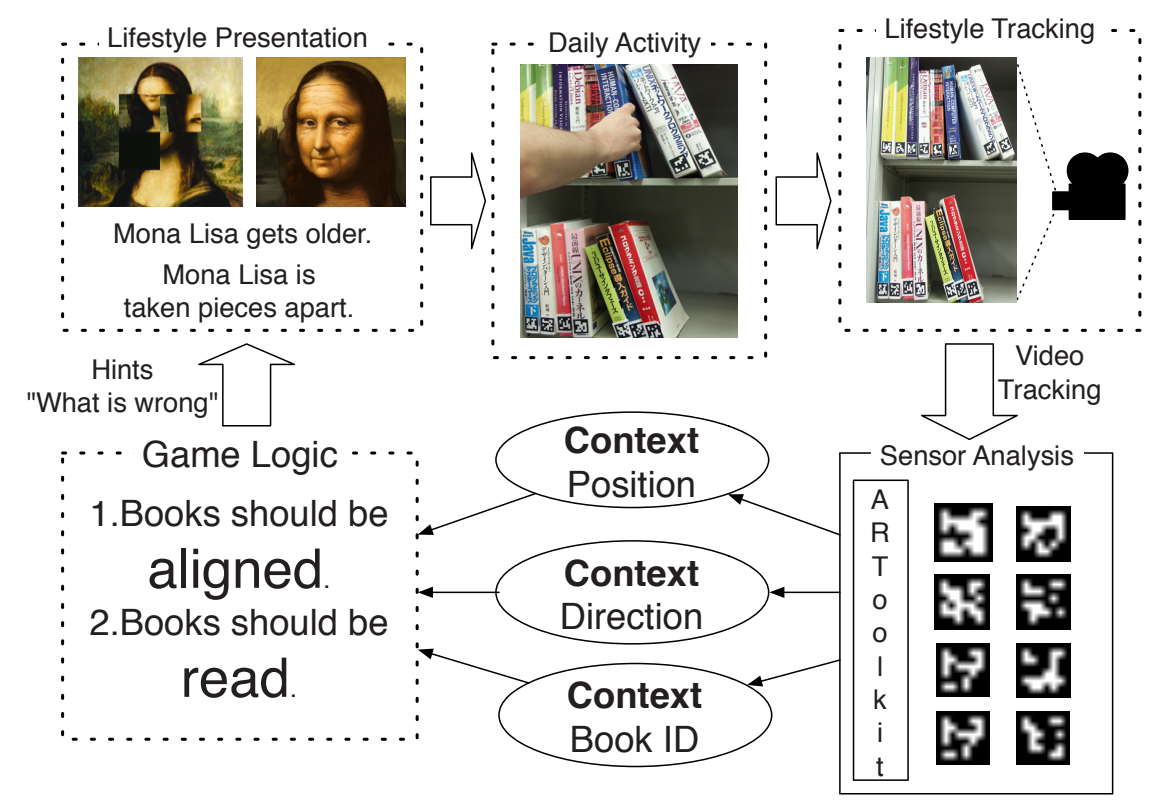

Fig. 5. Mona Lisa breaks up when some books are taken off for a while. She gets older when the bookshelf in a long while.

elegant and the window becomes clean. At last he stops toothbrushing, then fish stop dancing and start to school slowly again.

Gradual feedback If a player succeeded to brush both in morning and night, one of the fish blows an egg in at midnight. The egg hardly catches out initially. If $\mathrm{s} /$ he could continue to brush everyday, the incubation ratio increases. If $\mathrm{s} / \mathrm{he}$ could toothbrush in a right way, such as brushing both of upper and lower teeth, a rare kind of fish might be born (Fig. 4).

Implementation: Fig. 2 shows the overall architecture of this game based on our framework. The toothbrushing activity is tracked by Cookie sensor 12 that is a bluetooth-enabled coin-size sensor node having several kinds of sensors inside. This game uses a 3-axis accelerometer in it. One Cookie is attached to a toothbrush and the sensor analysis module analyzes accelerometer data while a player is toothbrushing. Each Cookie has own unique ID that is used as user ID in this game. Toothbrushing time is recognized by monitoring the difference between maximum and minimum acceleration. Toothbrushing pattern is recognized by monitoring the direction of bristles. Bluetooth connection, context acquisition and event notification are managed by our context acquisition framework [4.

\subsection{A Virtual Fine Art Improving Book Arrangement Activity}

The second case study is a game encouraging book arrangement activity in a personal bookshelf, which is a kind of cleaning activities. This game is based 

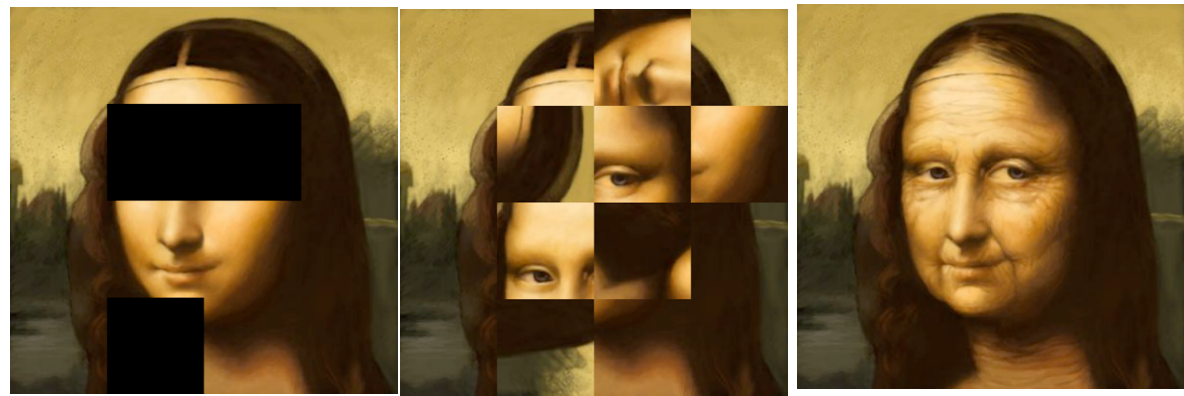

Fig. 6. Partly dropped Fig. 7. Broken Mona Lisa Fig. 8. Old Mona Lisa inMona Lisa indicating indicating books are not in dicating no books are read some books are not in the order recently bookshelf

on two rules that are Books should be arranged and aligned correctly and Books should be read at least once a weak or some span. It tracks the state of books in a bookshelf and provides immediate feedback for encouraging book arrangement and gradual feedback for encouraging reading books. These feedback are represented on a virtual fine art mounted near the bookshelf.

Immediate feedback: When a book is taken off for a while, a piece of Mona Lisa is also taken off. If books are not aligned correctly, the pieces of Mona Lisa are also aligned awkwardly. While the books are arranged correctly in the bookshelf, she smiles peacefully (Fig. 6, 7).

Gradual feedback: If non of the books are not read for a long while, she gets older slowly. Then, when one of the books is read, she gets back her youth and smile. Too old Mona Lisa indicates the books on the bookshelf should be replaced with other ones (Fig. 8).

Implementation: Fig. 5 shows the overall architecture similar in the first one. The positions of books are tracked by using of ARToolkit [10 that is a software library implementing visual tag recognition. A video camera deployed in front of a bookshelf captures visual tags attached to each book. Then, the analysis module calculates their positions, directions, and IDs and notifies them to the game logic. The logic gives hints including information of missing or unaligned books and unread books to the presentation module implemented using Flash.

\section{Discussion and Future Work}

In this section, we describe some discussions and future work given through designing and implementing prototype games described in case studies. 
Immediate feedback vs gradual feedback: We believe lifestyle ubiquitous games should have both of immediate and gradual feedback as described in our case studies. Immediate feedback is useful for notifying players the state of current tracking and making the moment of activities fun. Gradual feedback is appropriate for presenting them the history of tracked activities and encouraging the continuance of daily activities. We expect to observe players' behavior within each feedback result and prospect about relationship between them.

Direct vs indirect metaphor mapping: In our current case studies, the presentations like an aquarium and a fine art have few relevance with the actual activities like toothbrushing and book arrangement. That is because we thought if it is directly connected like the toothbrushing game shows deformed bad teeth, players who are lazy for toothbrushing might not be interested in it. However, that toothbrushing game encourages players who are already interested in toothbrushing more. We expect to build another game that presentation metaphor is directly mapped to the activity and prospect the difference.

User studies: We still have not conducted any formal user studies. We believe that we have to answer two questions: (1) Is it fun or not? How people accept or reject our gaming concept? (2) Can these games really encourage boring daily activities? For answering the first question, we expect to use think aloud technique and interview with short-term experiments. For the second one, we expect to conduct long-term experiments with following steps. At first, we observe subjects doing particular activities without games several days. Then, we deploy games in their home and observe changes in the activities several days. At last, we remove the games and continue to observe changes. Each observation can be easily done by using sensors embedded in daily objects.

Networking lifestyle ubiquitous gaming: Our prototype games are all stand-alone programs. It is because we thought that it was appropriate to explore lifestyle ubiquitous gaming with building simple games at first step. We identify that networking features can make our games much more fun. For example, competition of virtual aquariums' daily snapshots would make players more competitive. Daily activities on the Web such as blogging and social networking services could be easily integrated with lifestyle ubiquitous gaming like BlogPet[1].

Expected limitations of lifestyle ubiquitous gaming: The final question that we have to explore is "Can lifestyle ubiquitous games become current Hollywoodlike large-scale game alternatives?" We expect that even if we could achieve making quite attractive gaming world around lifestyle ubiquitous games, they can hardly become the alternatives for hard core gamers who truly love to play large scale games. But, we can see large movements in which ordinary people leave large scale console games to simple and convenient mobile games around in many parts of the world. We believe that exploring this research makes new alternatives for people who is hesitative about playing computer games. 


\section{Related Work}

Digital Game-Based Learning[14] discussed how learners have changed, how games teach and why they work. 3] and [1] described about serious games that are computer and video games that are intended not only entertain users, but have additional purposes such as education and training. As these books shows, the positive effects of games on learning, training, exercising, and medical things are being explored in recent years. However, how integrating gaming features into daily activities effects is not explored.

Several recent gaming projects so-called pervasive gaming [13] have exploited the possibility to combine the world of the game with the physical world. Pirates! 2 allows players to move around in the physical domain and are presented with location dependent games on their PDAs equipped with simple short-range radio frequency proximity sensors. Can You See Me Now?[5] combines a game of tag in a real city in online game-play in a virtual game area by connecting online players' home computers to real runners' handheld computers. These games show positive possibilities and ways to integrate computer games into physical domain, but still they require complicated configuration settings and significant time to enjoy.

FantasyA and SenToy 9 represent an affective control toy. SenToy is a doll including several sensors wirelessly connected to a PC. By using gestures of the doll, it allows players to manipulate the emotions of a synthetic character in the computer game, FantasyA. Physical user interfaces embedded within everyday objects like a SenToy doll would make it easy to entry virtual gaming worlds. But, doll gestures are probably not appropriate for adults and not related to daily activities. We believe that if we can embedded same kind of physical user interfaces of computer games into more common daily activities, that would make more intuitive and easy-to-entry virtual gaming worlds.

Informative art project [8] explores information visualization techniques that use art as inspiration for both their appearance and their role in our surroundings. Its information presentations disappear into the background and enable people to be notified information without too much attention. Lifestyle presentation in lifestyle ubiquitous gaming is a bit similar to informative art in terms of embedding information visualization in daily objects. But our idea includes purposeful movements like making boring tasks fun rather than just showing information.

\section{Conclusion}

In this paper, we showed a concept and case studies of lifestyle ubiquitous gaming that is a novel computer gaming style embedded in our daily lives. It makes boring daily activities fun and does not require too much attention and time to play. We believe this novel gaming idea can inspire the current game industry and open up new breakthroughs. But, currently this research is in the very early stage so that we still could not verify the effectiveness and feasibility. We 
are planning to do practical and long-term user studies and build more games exploring the other daily activities: cooking and resource saving.

\section{Acknowledgement}

This work is greatly supported by the Microsoft Institute for Japanese Academic Research Collaboration (IJARC) with universities. The authors would like to thank Ying-Qing Xu and Hajime Wada. The prototype games were developed by several students in our laboratory. The authors would like to thank Keisuke Hayashi, Yuri Kuno, Teruhide Kusaka, Hanae Suzuki, Chihiro Takayama and Keita Yamada.

\section{References}

1. Bergeron, B.: Developing Serious Games. Charles River Media (2006)

2. Björk, S., Falk, J., Hansson, R., Ljungstrand, P.: Pirates! using the physical world as a game board. In: Interact 2001, IFIP TC.13 Conference On Human-Computer Interaction, Tokyo, Japan (July 2001)

3. Chen, S.: Serious Games: Games That Educate, Train, and Info. Course Technology (2005)

4. Nakajima, T., Kawsar, F., Fujinami, K.: Prottoy: A middleware for sentient environment. In: Yang, L.T., Amamiya, M., Liu, Z., Guo, M., Rammig, F.J. (eds.) EUC 2005. LNCS, vol. 3824, Springer, Heidelberg (2005)

5. Flintham, M., Benford, S., Anastasi, R., Hemmings, T., Crabtree, A., Greenhalgh, C., Tandavanitj, N., Adams, M., Row-Farr, J.: here on-line meets on the streets: experiences with mobile mixed reality games. In: CHI '03. Proceedings of the SIGCHI conference on Human factors in computing systems, Ft. Lauderdale, Florida, USA, ACM Press, New York (2003)

6. Fujinami, K., Kawsar, F., Nakajima, T.: Awaremirror: A personalized display using a mirror. In: The 3rd International Conference on Pervasive Computing, Munich, Germany (May 2005)

7. Fujinami, K., Nakajima, T.: Sentient artefacts: Acquiring user's context through daily objects. In: The 2nd International Workshop on Ubiquitous Intelligence and Smart Worlds, Nagasaki, Japan (December 2005)

8. Holmquist, L.E., Skog, T.: Informative art: information visualization in everyday environments. In: GRAPHITE '03. Proceedings of the 1st international conference on Computer graphics and interactive techniques in Australasia and South East Asia, Melbourne, Australia (2003)

9. Höök, K., Bullock, A., Paiva, A., Vala, M., Chaves, R., Prada, R.: Fantasya and sentoy. In: CHI '03. CHI '03 extended abstracts on Human factors in computing systems, Ft. Lauderdale, Florida, USA, ACM Press, New York (2003)

10. Kato, H., Billinghurst, M.: Marker tracking and hmd calibration for a video-based augmented reality conferencing system. In: IWAR 99. Proceedings of the 2nd International Workshop on Augmented Reality, San Francisco, USA (October 1999)

11. KDDI CORPORATION and WORK@ INC. BlogPet.http://www.blogpet.net/ Last checked: (July 24, 2006) 
12. Kimura, H., Tokunaga, E., Okuda, Y., Nakajima, T.: Cookieflavors: easy building blocks for wireless tangible input. In: CHI '06. CHI '06 extended abstracts on Human factors in computing systems, Montréal, Québec, Canada (2006)

13. Magerkurth, C., Cheook, A.D., Mandryk, R.L., Nilsen, T.: Pervasive games: Bringing computer entertainment back to the real world. ACM Computers in Entertainment 3(3) (2005)

14. Prensky, M.: Digital Game-Based Learning. McGraw-Hill Companies, New York (2000) 